\title{
Delta Tocotrienol and Melatonin in Management of Mast Cell Canine Tumor
}

\author{
Fabio Olivieri* \\ Clinical Research Unit, EFFEGILAB S.r.l., Trento, Italy \\ *Corresponding author: Fabio Olivieri, Clinical Research Unit, EFFEGILAB S.r.l., Trento, Italy
}

\section{ARTICLE INFO}

Received: 蔧 June 26, 2020

Published: 慧 July 06, 2020

Citation: Fabio Olivieri. Delta Tocotrienol and Melatonin in Management of Mast Cell Canine Tumor. Biomed J Sci \& Tech Res 28(4)-2020. BJSTR. MS.ID.004690.
ABSTRACT

Background and Objectives: The antineoplastic effects of Vitamin E and its derivatives, Tocopherols and Tocotrienols are known in scientific literature [1-3]. In particular, it has been shown that the fractions of Tocotrienol $(\alpha, \beta, \gamma, \delta)$ are active on different types of cancer, reducing their growth through different pathways [4-6]. Also, Melatonin is known as a molecule capable of regulating the rate of cell growth, with particular activity on cancer cells through different pathways [7-9]. In this study we observed how the dog's mastocytoma was managed by associating the Delta Tocotrienol with Melatonin conjugated with Adenosine and Glycine.

Methods: Eight dogs were treated from May 2018 to June 2019 with Delta Tocotrienol (TOCOTRIENOL $\mathrm{FG}^{\circledR}$ ) and the Melatonin-Adenosine-Glycine complex (MELATONINA COMPLEX ${ }^{\circledR}$ ). The diagnosis in all eight cases was I, II and III-degree mastocytoma with no metastasis. The dogs were tested for biochemical and blood count tests and evaluated clinically every two months.

Result: During the treatment period, only one of the dogs with trunk nodule continued to have relapses after surgery, although less frequently than before treatment, and with a slower evolution. None of the patients had been treated with chemotherapy by the owners' will.

Conclusion: Delta Tocotrienol and the Melatonin-Adenosine-Glycine complex allowed the control of tumor development, a reduction of post-surgical recurrences and the total absence of side effects. After a year of treatment, no dog developed mastocytoma again.

\section{Case Report}

Mast Cell Tumor in dogs is one of the most frequent malignant tumors. Often, the pet owner categorically refuses the use of chemotherapy, asking for a manteinance therapy that could improve or stabilize the status health of the own pet. Currently there are several plant extracts and natural molecules that have been shown to possess antineoplastic activity in the scientific literature. Among these, Tocotrienols and Melatonin have shown in vitro and in vivo some activities on many types of tumors. Eight dogs with mastocytoma have been treated with these two molecules for one year.

Mastocytoma is one of the most common cancers in dogs [10]. It originates from mast cells, cells abundantly present in the connective tissue and involved in anaphylactic, allergic and hypersensitivity reactions and which occur during inflammation.
They contain material intended to be expelled during trauma, infection and inflammation, in particular serotonin, NGF, histamine and heparin. Mast cell tumor is characterized by a rather varied biological behavior. In most cases it is limited to a localized, nodular and isolated form, without giving any symptoms either local or systemic, other times it can develop as a multinodular, systemic and aggressive disease (11-14\%). Generally, these lesions appear as nodular masses, not always pedunculated, often ulcerated, alopecic and erythematous, present at the level of the dermis or subcutaneous tissue. They can appear as isolated or multicentric formations and it is also possible that metastasis occurs to the lymph nodes or other organs [11]. For these reasons, skin nodules could be often associated with generalized clinical signs, such as vomiting, anorexia, ulcerative gastritis, coagulation disorders, due to a massive release of vasoactive substances by mast cells. 
Its etiopathogenesis is not yet known there is likely to be a genetic mutation underlying the development of this tumor as some breeds are more predisposed than others. Among the breeds that are most affected by this pathology are Boxer, Bull Terrier, English Bulldog, Beagle, Shar-pei (the latter even at a young age), Labrador and Golden Retriever. At the clinical visit the general conditions of the patients were on average good, the blood chemistry parameters did not indicate particular out-of-scale values, with the exception of a hypothyroid castrated bitch, treated contextually with Levothyroxine. Five dogs (two female Labrador, three Boxer, one female two males) had a grade 1 mastocytoma. In these five group, two Labrador had paw injury, two Boxer males had scrotal nodules and the female Boxer had tail nodule. Two half breed male dogs had grade 2 trunk lesions, one female dog (Golder Retriever) had grade 3 ulcerated right paw. None of the lesions exceeded $15 \mathrm{~mm}$ in diameter

In three dogs (two boxer and Golden Retriever) there was the "sign of Darier", indicative and pathognomonic of mast cell tumor. The diagnosis was made in all cases by needle aspiration cytological examination. no dog had metastasis, checked by ultrasound and radiography. The average weight was $28 \mathrm{Kg}$ (24 to $32 \mathrm{~kg}$ ). All dogs were treated with Delta Tocotrienol $150 \mathrm{mg}$ BID and Melatonin (with Adenosine-Glycine) $4 \mathrm{mg}$ BID five days a week for 3 months at a time with two weeks of suspension. In seven dogs in which the nodules had been surgically removed, relapses never occurred again, only in one dog did a relapse occur. The nodules that have not been removed due to anatomical problems have significantly reduced in volume and many of which have disappeared within the first three months of treatment. No dog had any side effects related to the administration of the two food supplements.

\section{Conflicts of Interest}

The authors have no conflicts of interest to declare.

\section{References}

1. Tsuyoshi Tsuduki, Keiko Kuriyama, Kiyotaka Nakagawa, Teruo Miyazawa (2013) Tocotrienol Unsaturated Vitamin E) Suppresses Degranulation of Mast Cells and Reduces Allergic Dermatitis in Mice. J Oleo Sci 62(10): 825-834.

ISSN: $2574-1241$

DOI: $10.26717 /$ BJSTR.2020.28.004690

Fabio Olivieri. Biomed J Sci \& Tech Res

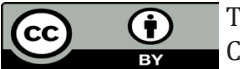

This work is licensed under Creative Commons Attribution 4.0 License

Submission Link: https://biomedres.us/submit-manuscript.php
2. Rony A Francois, Anying Zhang, Kazim Husain, Chen Wang, Sean Hutchinson, et al. (2019) Vitamin E $\delta$-Tocotrienol Sensitizes Human Pancreatic Cancer Cells to TRAIL-induced Apoptosis Through Proteasome-Mediated Down-Regulation of c-FLIP s. Cancer Cell Int 19: 189.

3. Fabrizio Fontana, Roberta Manuela Moretti, Michela Raimondi, Monica Marzagalli, Giangiacomo Beretta, et al. (2019) $\delta$-Tocotrienol Induces Apoptosis, Involving Endoplasmic Reticulum Stress and Autophagy, and Paraptosis in Prostate. Cancer Cells Cell Prolif 52(3): e12576.

4. Qing Jiang (2019) Natural Forms of Vitamin E and MetabolitesRegulation of Cancer Cell Death and Underlying Mechanisms. IUBMB Life 71(4): 495-506.

5. Chao Yang, Qing Jiang (2019) Vitamin E $\delta$-Tocotrienol Inhibits TNF- $\alpha$ stimulated NF-kB Activation by Up-Regulation of Anti-Inflammatory A20 via Modulation of Sphingolipid Including Elevation of Intracellular Dihydroceramides. J Nutr Biochem 64: 101-109.

6. Lichchavi D Rajasinghe, Rohini H Pindiprolu, Smiti Vaid Gupta (2018) Delta-tocotrienol Inhibits Non-Small-Cell Lung Cancer Cell Invasion via the Inhibition of NF-kB, uPA Activator, and MMP-9. Onco Targets Ther 11:4301-4314.

7. Haijuan Gu, Qian Shen, Dan Mei, Yang Yang, Rong Wei, et al. (2020) Melatonin Inhibits TE-1 Esophageal Cancer Cells Metastasis by Suppressing the NF-kB Signaling Pathway and Decreasing MMP-9. Ann Clin Lab Sci 50(1): 65-72.

8. Xueran Chen, Aijun Hao, Xian Li, Zhaoxia Du, Hao Li, et al. (2016) Melatonin Inhibits Tumorigenicity of Glioblastoma Stem-Like Cells via the AKT-EZH2-STAT3 Signaling Axis. J Pineal Res 61(2): 208-217.

9. Peng Liu, Xinhua Xie, Anli Yang, Yanan Kong, Diane Allen-Gipson, et al. (2020) Melatonin Regulates Breast Cancer Progression by the lnc010561/miR-30/FKBP3 Axis. Mol Ther Nucleic Acids 19: 765-774.

10. Elisa Baioni, Eugenio Scanziani, Maria Claudia Vincenti, Mauro Leschiera, Elena Bozzetta, et al. (2017) Estimating canine cancer incidence: findings from a population-based tumour registry in northwestern Italy. BMC Vet Res 13: 203

11.EL Krick, AP Billings, FS Shofer, S Watanabe, KU Sorenmo (2009) Cytological Lymph Node Evaluation in Dogs with Mast Cell Tumours: Association with Grade and Survival. Vet Comp Oncol 7(2): 130-138.

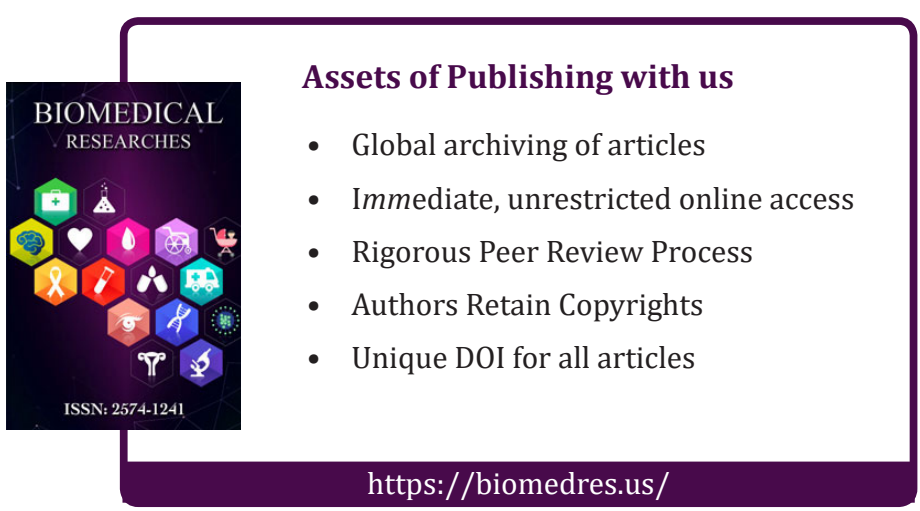

Copyright@ Fabio Olivieri | Biomed J Sci \& Tech Res | BJSTR. MS.ID.004690. 
